\title{
A Fourteen-Year Review of Radical Cystectomy for Transitional Cell Carcinoma Demonstrating the Usefulness of the Concept of Lymph Node Density
}

\author{
Chi W. Cheng, Chi F. Ng, Chi K. Chan, Wai S. Wong, Pun E. Hui, Yim F. Wong \\ Department of Surgery and Department of Clinical Oncology, The Chinese University of Hong \\ Kong, Prince of Wales Hospital, Hong Kong, China
}

\begin{abstract}
Objective: We studied the long-term outcome of radical cystectomy for transitional cell carcinoma and evaluated prognostic factors for disease specific survival.

Materials and Methods: A retrospective review was carried out for all cystectomies performed for transitional cell carcinoma between 1989 and 2002. Disease specific survival was correlated to patient, pathological and operative factors as well as to adjuvant therapy.

Results: Of the 133 cystectomies included, 100 were male and 33 were female patients. The median age was 69 years (range 43 to 86). The median follow up was 20 months (range 0 to 158). With univariate analysis, pT stage, $\mathrm{N}$ stage, lymph node density, carcinoma in-situ, surgical margin and post-operative radiotherapy to distant metastasis were predictive of disease specific survival. On the other hand, with multivariate analysis, only pT stage, lymph node density and postoperative radiotherapy to distant metastasis were predictive of disease specific survival. Within the group of node positive disease, lymph node density also predicted disease specific survival with both univariate and multivariate analyses. Patients with lymph node density $20 \%$ or below showed better disease specific survival.

Conclusions: pT stage and lymph node density were found to be the most important predictive factors for disease specific survival after cystectomy in the Asian population.
\end{abstract}

Key words: bladder; carcinoma, transitional cell; cystectomy; lymph nodes

Int Braz J Urol. 2006; 32: 536-49

\section{INTRODUCTION}

Radical cystectomy is the standard surgical treatment for muscle invasive bladder transitional cell carcinoma (TCC) and can achieve good long term results for organ confined disease. Patients with locally advanced or nodal disease may also benefit from the surgery (1-3). pT staging and N staging have been widely accepted as important prognostic factors.
Both the value and the extent of pelvic lymphadenectomy have been controversial. Lymph node density (LND), defined as the percentage of resected lymph nodes that were involved and calculated by the division of the number of lymph nodes involved by the number resected, has also been found to be a useful prognostic variable (4). A retrospective review of radical cystectomy was carried out to evaluate prognostic factors including the LND 
to see if they were also applicable to the Asian population.

\section{MATERIALS AND METHODS}

Radical cystectomy performed in the Prince of Wales Hospital for TCC between 1989 and 2002 were reviewed. Cystectomy was preceded by bilateral pelvic lymphadenectomy with the two specimens sent separately and followed by either an ileal conduit or a continent diversion. The boundaries of the pelvic lymphadenectomy were the muscle pelvic side wall laterally, vesical fascia medially, lateral border of external iliac vein superiorly, obturator nerve inferiorly, bifurcation of the common iliac artery proximally and femoral canal inferiorly.

Patients with bulky tumors might receive radical radiotherapy (RT) before salvage cystectomy. Younger patients with good renal function might receive neoadjuvant systemic chemotherapy before radical cystectomy at the discretion of the urologists. Patients with positive pelvic lymph nodes or minimal or positive surgical margins might receive postoperative RT to the pelvis. Patients with recurrence as distant metastases might receive post-operative systemic chemotherapy or post-operative RT to the metastases at the discretion of the oncologists.

Data were collected from patient case notes, patient or family telephone contact and electronic medical records, including patient (age, sex, mode of presentation), pathological (pT stage, $\mathrm{N}$ stage, number of nodes involved, number of nodes resected, LND, grading, coexistence of carcinoma in-situ or CIS, surgical margin) and operative factors (continent diversion, simultaneous nephrectomy, simultaneous urethrectomy) as well as adjuvant therapy (RT, chemotherapy). The staging system employed was the 2002 American Joint Committee on Cancer TNM staging system. In cases of mixed stages or grades, the highest stage or grade was documented. Presentation mode could either be progression from superficial disease or de novo muscle invasive. LND was defined as stated above. A positive surgical margin means a positive one confirmed microscopically and a minimal margin dictates that cancer cells are found $1 \mathrm{~mm}$ or less close to the surgical margin.

End points recorded included recurrence and death. The time to first recurrence, the survival and the cause of death were documented. Recurrence free interval and patient survival were defined as the time from cystectomy to the end point (recurrence, death or censored). In the analysis of disease specific survival, patients who died of TCC were classified as deaths.

The Kaplan-Meier method was used to calculate the survival curves. The log rank test and the Cox proportional hazards model were used for univariate and multivariate analyses respectively, to assess the influence on the survival curves by patient, pathological and operative factors as well as adjuvant therapy. Logistic regression was used to assess the influence on LND by pre-operative and intraoperative parameters.

\section{RESULTS}

\section{Clinico-pathological Features}

One hundred and forty four cystectomies were performed in the 14 year period. Eleven cases were excluded from this review because of histology other than TCC (seven cases of adenocarcinoma, one case of squamous cell carcinoma, one case of leiomyosarcoma and two cases of carcinosarcoma). There were 100 males and 33 females. The mean and median ages were 67.1 and 69 years (range 43 to 86), respectively.

The indications for radical cystectomy were mainly muscle invasive disease (123 cases) except one case of CIS resistant to treatment and nine cases of recurrent pT1 disease. The details of histological findings were shown in Table-1. Reconstruction was achieved by ileal conduit in 112 cases and continent diversion in 21 cases. Continent diversion performed included 19 ileal neobladders, one Indiana pouch and one right colonic pouch. Nine patients had a simultaneous nephrectomy (three cases for simultaneous upper tract TCC and six cases for nonfunctioning kidneys) and 25 patients had a simultaneous urethrectomy. Lymph node status was 
Table 1 - Number of patients with different histological findings.

\begin{tabular}{|c|c|c|}
\hline \multirow[t]{3}{*}{ Mode of presentation } & Progression from superficial & 14 \\
\hline & De novo muscle invasion & 109 \\
\hline & Non-muscle invasive at cystectomy & 10 \\
\hline \multirow[t]{10}{*}{ pT stage } & CIS & 1 \\
\hline & 1 & 9 \\
\hline & 2 & 14 \\
\hline & $2 \mathrm{a}$ & 25 \\
\hline & $2 b$ & 16 \\
\hline & $3 a$ & 17 \\
\hline & $3 b$ & 25 \\
\hline & $4 \mathrm{a}$ & 22 \\
\hline & $4 \mathrm{~b}$ & 4 \\
\hline & Missing data & 0 \\
\hline \multirow[t]{5}{*}{$\mathrm{N}$ stage } & NO & 82 \\
\hline & $\mathrm{N} 1$ & 12 \\
\hline & $\mathrm{N} 2$ & 25 \\
\hline & $\mathrm{N} 3$ & 1 \\
\hline & Missing data & 13 \\
\hline \multirow[t]{3}{*}{ M stage } & M0 & 132 \\
\hline & M1 & 1 \\
\hline & Missing data & 0 \\
\hline \multirow[t]{7}{*}{ Grading } & G1 & 1 \\
\hline & $\mathrm{G} 2$ & 25 \\
\hline & G3 & 106 \\
\hline & Missing data & 1 \\
\hline & CIS & 19 \\
\hline & Nil & 114 \\
\hline & Missing data & 0 \\
\hline \multirow[t]{4}{*}{ Lymph node density } & $0 \%$ & 82 \\
\hline & $20 \%$ or less & 15 \\
\hline & Above $20 \%$ & 22 \\
\hline & Missing data & 14 \\
\hline \multirow[t]{4}{*}{ Margin } & Clear & 105 \\
\hline & Minimal of $1 \mathrm{~mm}$ or less & 18 \\
\hline & Positive & 10 \\
\hline & Missing data & 0 \\
\hline
\end{tabular}

CIS = carcinoma in-situ.

unknown in thirteen patients, otherwise, thirty eight patients $(38 / 120=31.7 \%)$ showed positive lymph nodes. The mean and median numbers of nodes resected for the whole series was 9.48 and eight, respectively (range 1 to 49 ) while the mean and median numbers of nodes involved was 0.93 and zero, respectively (range 0 to 13 ). On the other hand, the mean and median numbers of nodes resected for node positive cases was 9.62 and seven, respectively (range 1 to 49) while the mean and median numbers of nodes involved was 2.95 and two, respectively (range 1 to 13). In patients free of nodal disease, the mean and median numbers of nodes resected were 9.65 and eight, respectively (range 1 to 33). 
The distribution of various pathological subgroups was as follows: $7.5 \%$ were superficial lymph node negative, $35 \%$ were muscle invasive lymph node negative, $25.8 \%$ were extravesical lymph node negative and $31.7 \%$ were lymph node positive disease. Moreover, the incidence of nodal involvement in various pT stages was found to be $11.1 \%$ in pT1, $16.0 \%$ in $\mathrm{pT} 2,43.6 \%$ in $\mathrm{T} 3$ and $57.1 \%$ in $\mathrm{T} 4$.

The number of cases that received RT and chemotherapy are shown in Table- 2 .

Table 2 - Number of patients with radiotherapy or chemotherapy.

\begin{tabular}{ll}
\hline Preoperative radiotherapy to bladder & 5 \\
Postoperative radiotherapy to pelvis & 12 \\
Postoperative radiotherapy to distant metastasis & 21 \\
Preoperative chemotherapy & 16 \\
Postoperative chemotherapy & 5 \\
\hline
\end{tabular}

\section{Endpoints}

Sixty one cases recurred at a median surveillance of 15 months (range 0 to 158). The median time to recurrence was nine months (range 0 to 67). Four cases recurred locally while the remaining 57 recurred as distant metastases.

Eighty four cases died being 68 of cancer at a median follow up of 20 months (range 0 to 158). Five cases of perioperative mortality $(5 / 133=3.8 \%)$ within 30 days of cystectomy were also classified as cancer, as they died as a result of a procedure for the disease. The median time to cancer death was 13.5 months (range 0 to 118 ).

The 1,3,5 and 10-year overall survival were $69.5 \%, 47.4 \%, 37.2 \%$ and $25.2 \%$, while the corresponding disease specific survival were $74.1 \%$, $53.7 \%, 42.2 \%$ and $34.6 \%$. The corresponding survival curves were shown in Figure-1.

The 5 and 10-year disease specific survival for various pathological subgroups were as follows:

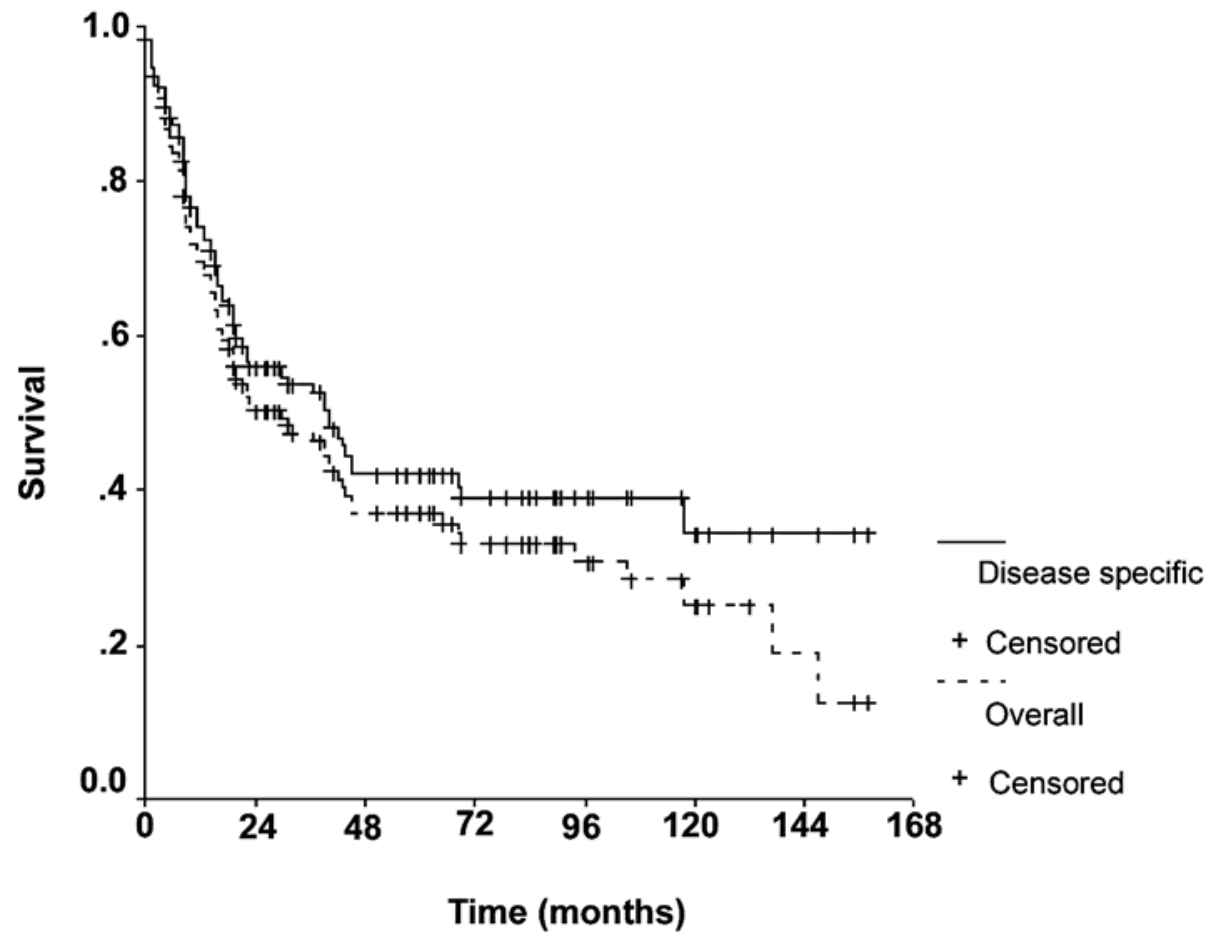

Figure 1-Overall and disease specific survival curves. 
Table 3 - Summary of univariate and multivariate analyses of effects of various prognostic factors on disease specific survival.

\begin{tabular}{llcc}
\hline & & Log Rank & Cox Regression \\
\hline \multirow{2}{*}{ Patient factors } & Sex & 0.4308 & 0.748 \\
& Age & & 0.193 \\
Pathological factors & Presentation & 0.5542 & 0.936 \\
& pT stage & $<0.0001^{*}$ & $0.003^{*}$ \\
& N stage & $0.0003^{*}$ & 0.847 \\
Nodes involved & & 0.838 \\
& Nodes resected & & 0.276 \\
LND & & $<0.001^{*}$ \\
Grading & CIS & $0.0001^{*}$ & 0.328 \\
Margin & 0.2912 & 0.987 \\
Continent diversion & $<0.0001^{*}$ & 0.147 \\
Nephrectomy & 0.5009 & 0.997 \\
& Urethrectomy & 0.1502 & 0.588 \\
& Preoperative chemotherapy & 0.5158 & 0.939 \\
& Preoperative RT & 0.2308 & 0.826 \\
& Postoperative chemotherapy & 0.5135 & 0.965 \\
& Postoperative RT to pelvis & 0.2894 & 0.236 \\
& Postoperative RT to metastasis & 0.0703 & 0.381 \\
& & $0.0001^{*}$ & $0.011^{*}$
\end{tabular}

$R T=$ radiotherapy; $*$ statistically significant.

$71.4 \%$ and $35.7 \%$ for superficial lymph node negative, $72.7 \%$ and $61.5 \%$ for muscle invasive lymph node negative, $19.9 \%$ and $19.9 \%$ for extravesical lymph node negative and $28.7 \%$ and $28.7 \%$ for lymph node positive disease.

\section{Prognostic Factors}

With univariate analysis, pT staging, $\mathrm{N}$ staging, LND, CIS, surgical margin and post-operative RT to distant metastasis were predictive of disease specific survival. With multivariate analysis, only $\mathrm{pT}$ staging, LND and post-operative RT to distant metastasis were predictive of disease specific survival.

The disease specific survival curves defined by those factors predictive of disease specific survival were shown from Figures-2 to 7. The results of univariate and multivariate analyses on disease specific survival for all factors were summarized in Table-3. The disease specific survival curves for various pT stages were shown in Figure-2. The 5- year survival for pT1, pT2, pT3 and pT4 disease were $71.4 \%, 69.1 \%, 17.2 \%$ and $12.2 \%$, respectively (log rank, $\mathrm{p}<0.0001)$. The disease specific survival curves for various $\mathrm{N}$ stages were shown in Figure-3. The 5year survival for $\mathrm{N} 0, \mathrm{~N} 1, \mathrm{~N} 2$, and $\mathrm{N} 3$ disease were $52.2 \%, 61.0 \%, 17.8 \%$ and $0 \%$, respectively (log rank, $\mathrm{p}=0.0003$ ). Similarly to what had been previously reported in literature, we also found a division line on LND at $20 \%$ discriminated patients into two groups with respect to disease specific survival (log rank, $p$ $<0.0001)$. The disease specific survival curves for LND of $20 \%$ or less and LND more than $20 \%$ were shown in Figure-4. 54.0\% of patients with LND of $20 \%$ or less survived five years or more, whereas all patients with LND more than $20 \%$ were either dead or censored at 39 months. The disease specific survival by surgical margin was shown in Figure-5. The 5-year disease specific survival for a clear, minimal or positive margin was $52.3 \%, 8.3 \%$ and $0 \%$ respectively (log rank, $\mathrm{p}<0.0001)$. The disease 


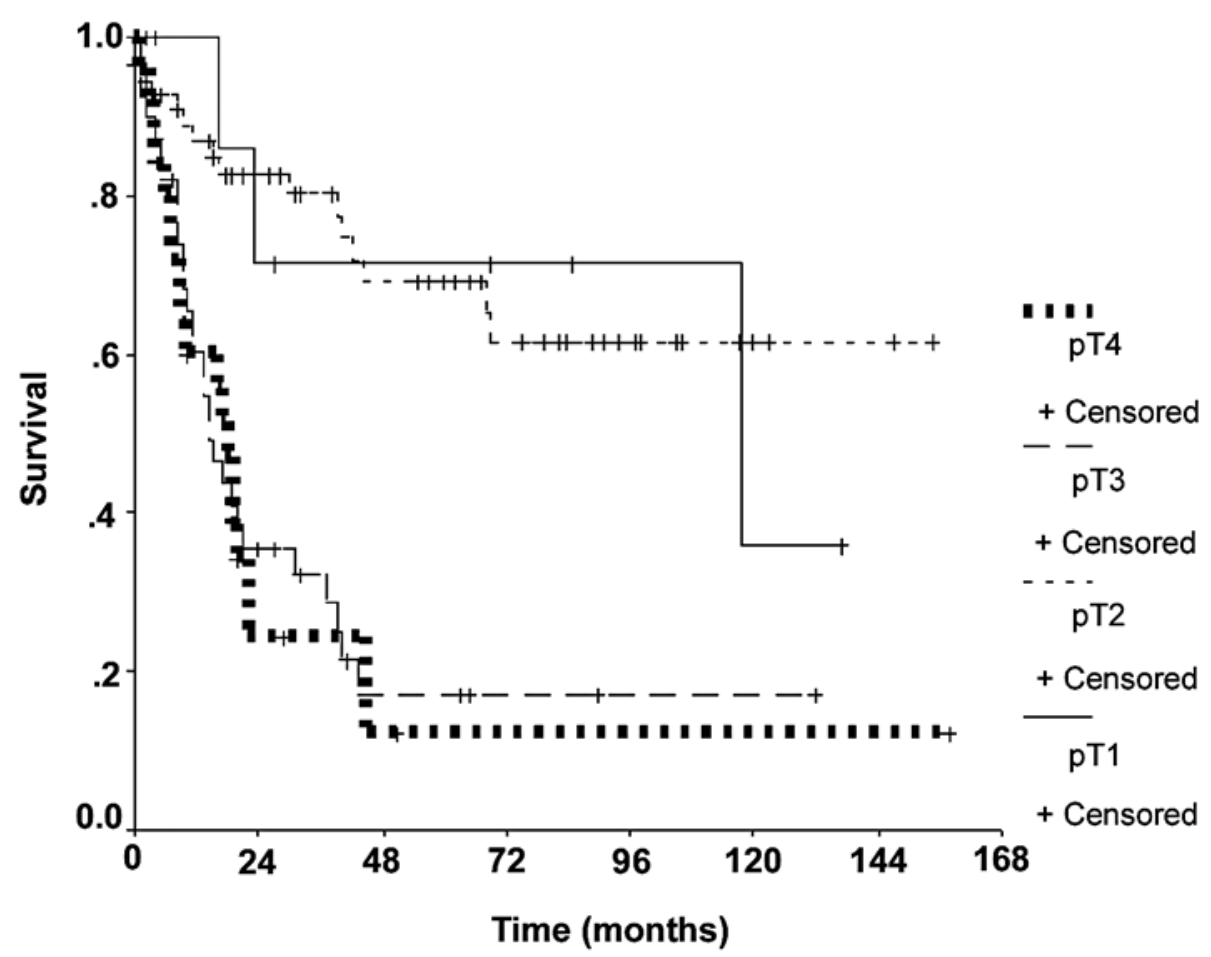

Figure 2 - Disease specific survival by pT stage.

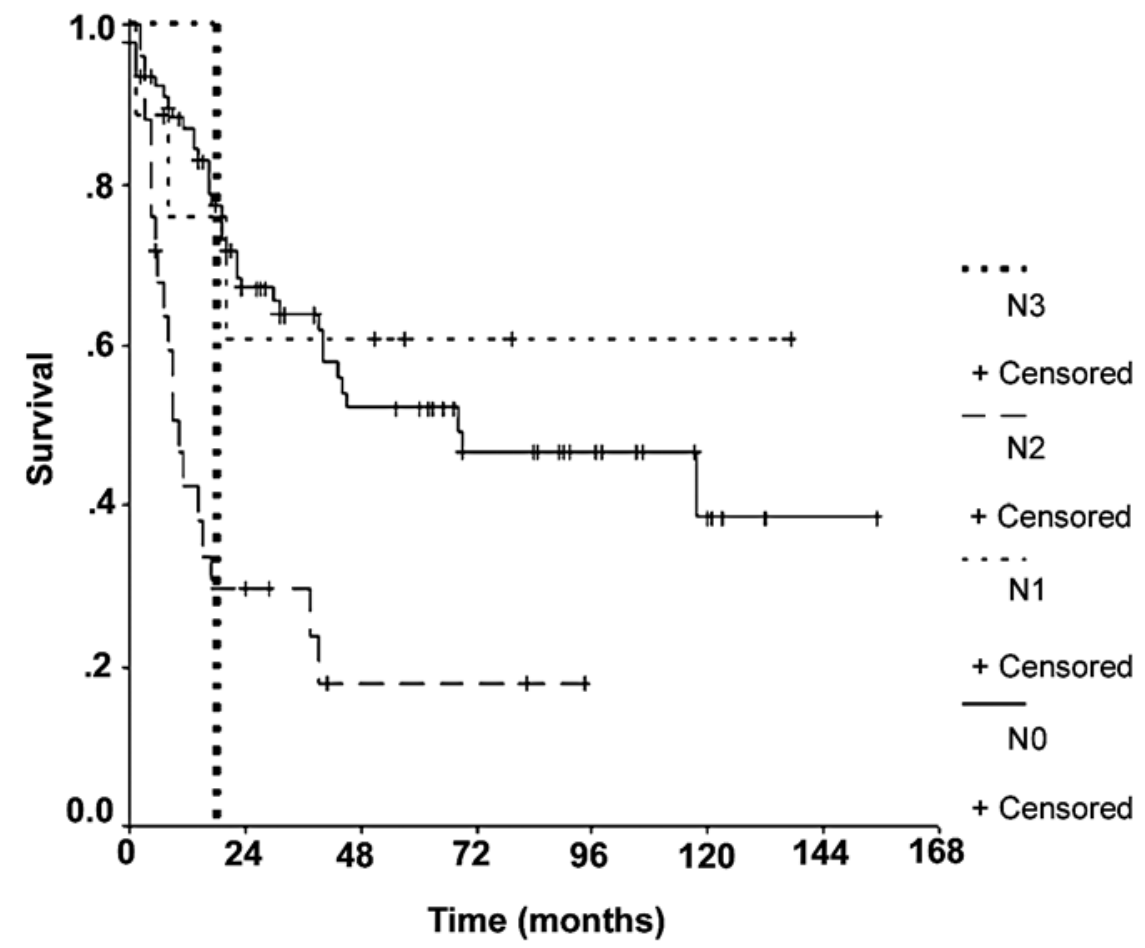

Figure 3 - Disease specific survival by $N$ stage. 


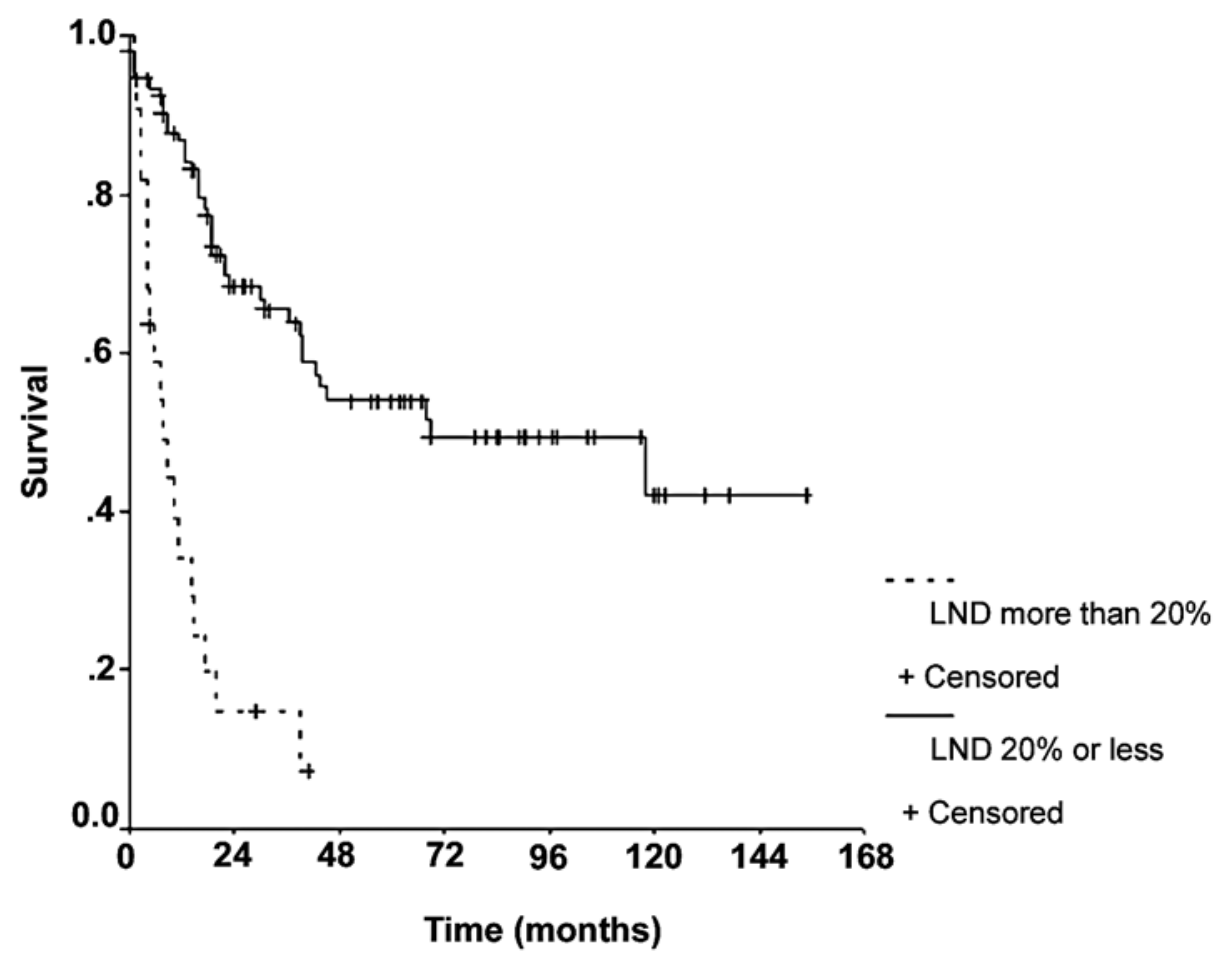

Figure 4-Disease specific survival by lymph node density.

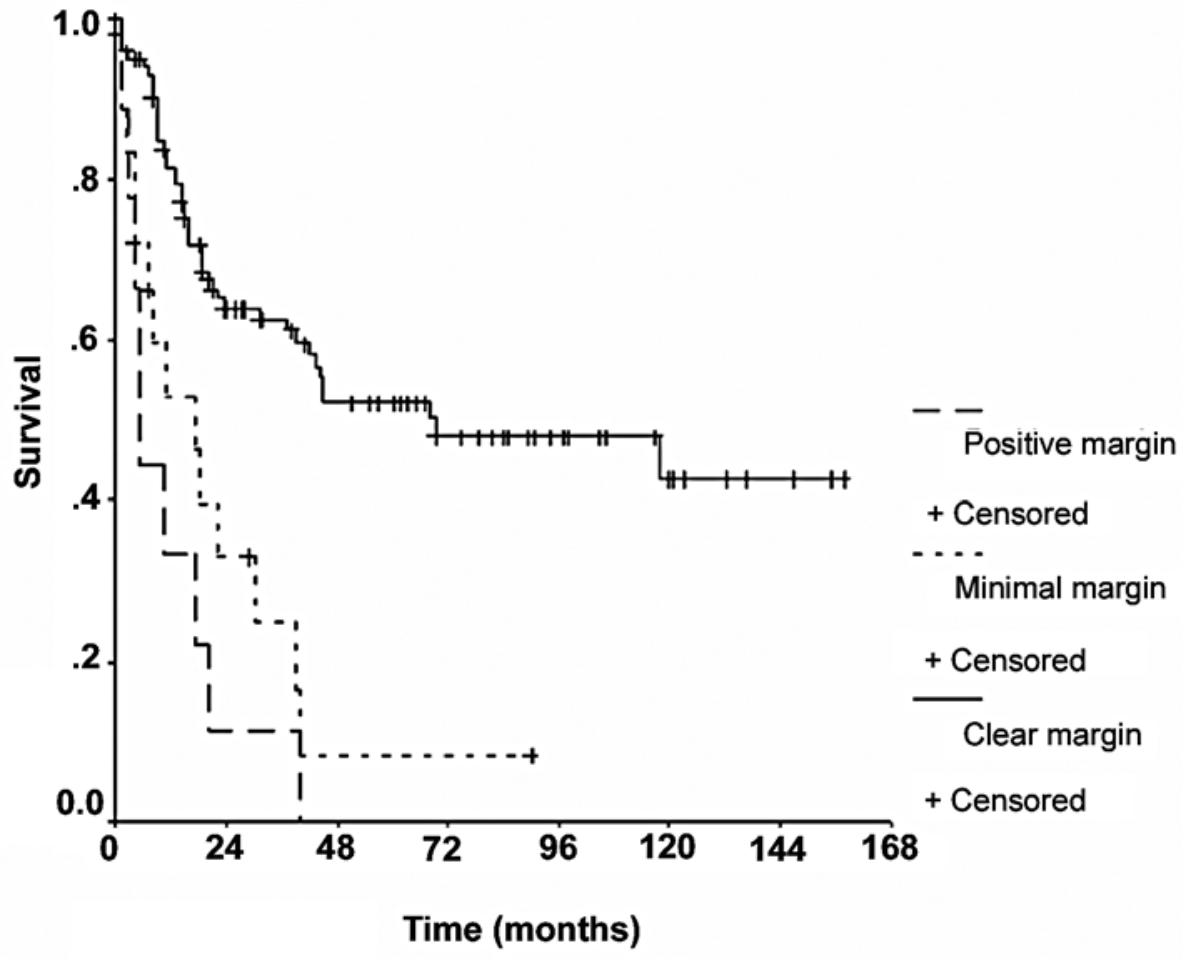

Figure 5 - Disease specific survival by surgical margin. 
specific survival by presence or absence of CIS was shown in Figure-6. The 5-year disease specific survival was $37.9 \%$ without CIS and $64.9 \%$ with CIS (log rank, $\mathrm{p}=0.0417$ ). The disease specific survival by post-operative RT to distant metastasis was shown in Figure-7. The 5-year disease specific survival was $51.9 \%$ without post-operative RT to distant metastasis and $0 \%$ with post-operative RT to distant metastasis (log rank, $\mathrm{p}=0.0001)$.

Within the group of node positive disease, we also found a division line on LND at $20 \%$ discriminated patients into two groups with respect to disease specific survival ( $\log$ rank, $\mathrm{p}=0.0003$ ). LND of $20 \%$ predicted disease specific survival with both univariate and multivariate analyses. The disease specific survival curves for LND of $20 \%$ or less and LND more than $20 \%$ in patients with nodal disease were shown in Figure-8. 64.3\% of patients with LND of $20 \%$ or less survived five years or more, whereas all patients with LND more than $20 \%$ were either dead or censored at 39 months. Furthermore, in this group of node positive patients, neither the pT stage nor the number of nodes involved or resected, affected the disease specific survival in multivariate analysis.

We also analyzed by logistic regression the effects on LND (cut off point at 20\%) by pre-operative and intra-operative factors to see if LND could be predicted before proceeding with cystectomy. These factors included sex, age, presentation mode, $\mathrm{pT}$ stage, grading, CIS, number of nodes resected, pre-operative RT, pre-operative chemotherapy, continent diversion, simultaneous nephrectomy and simultaneous urethrectomy. However, no such factor was found to predict LND in our series.

\section{COMMENTS}

This is a retrospective review with a relatively small sample size and short follow up period than

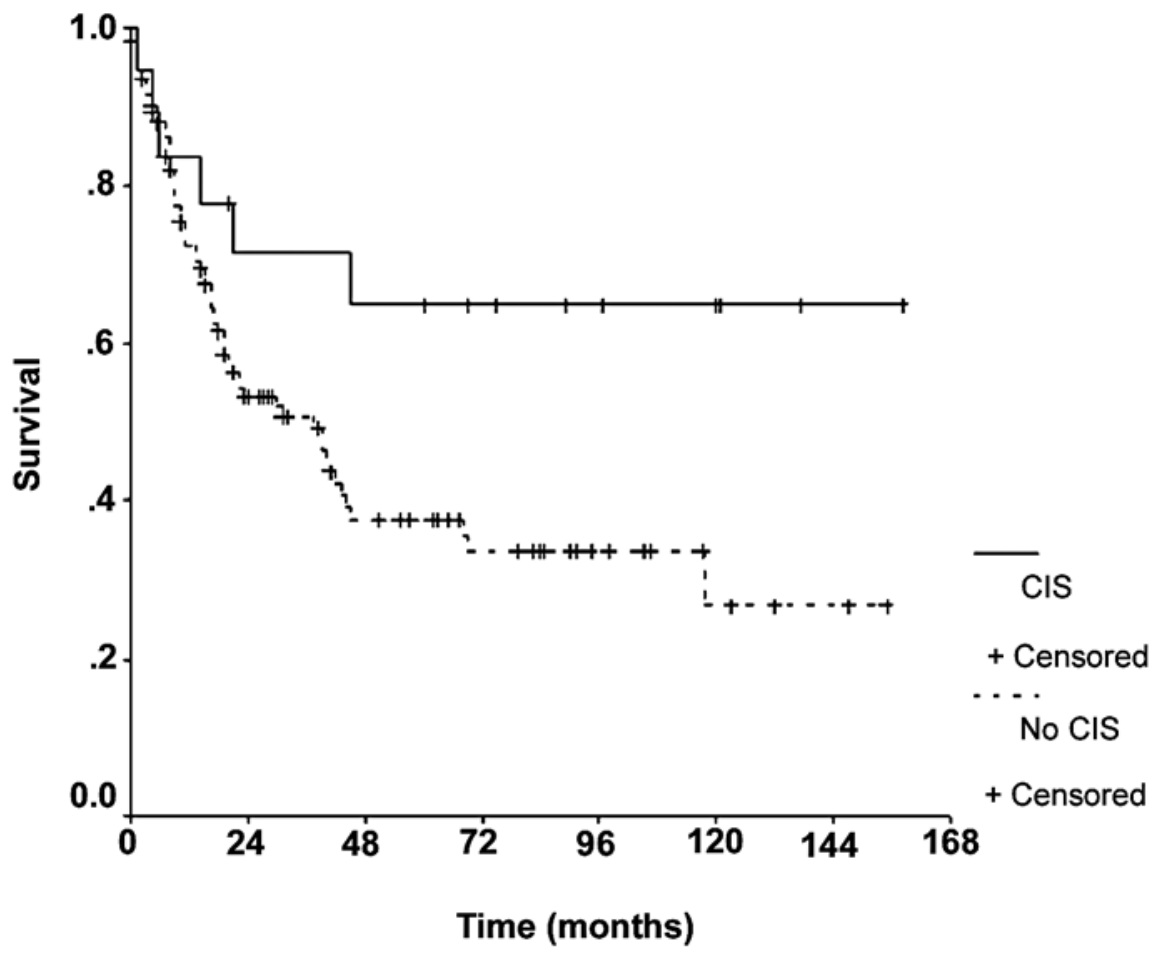

Figure 6-Disease specific survival by presence of carcinoma in-situ. 


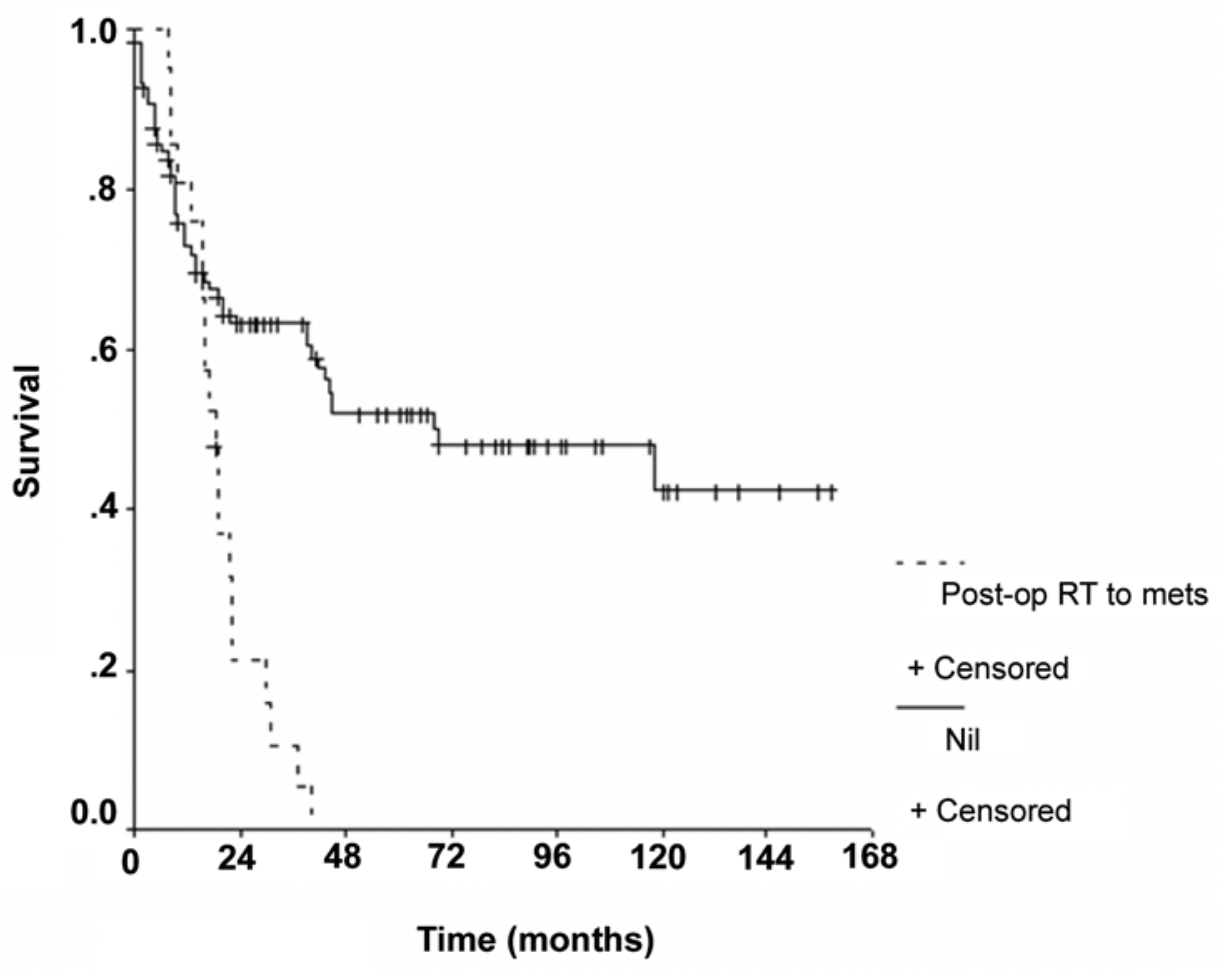

Figure 7 - Disease specific survival by postoperative radiotherapy to distant metastasis.

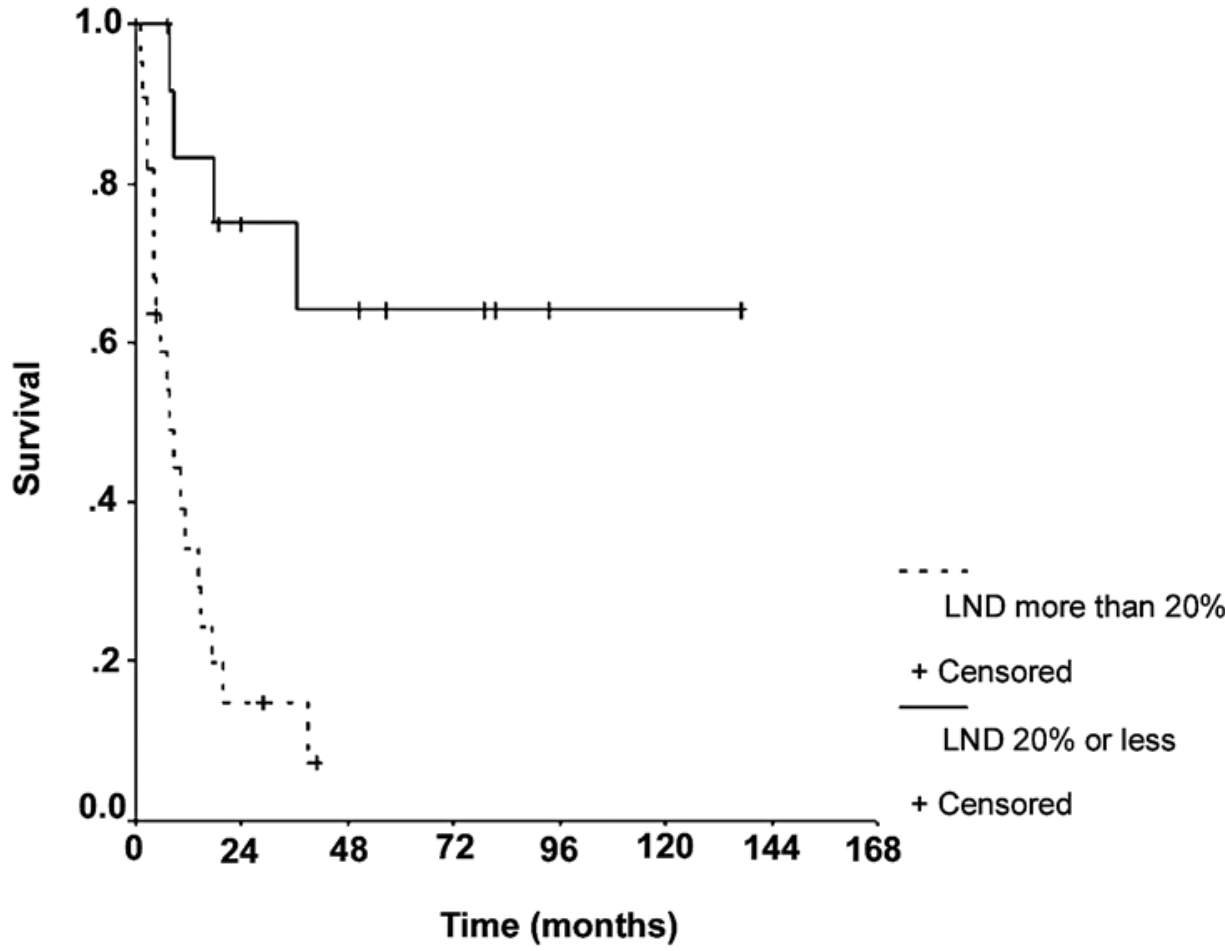

Figure 8-Disease specific survival by lymph node density in node positive patients. 
similar review in the literature. It is a heterogeneous group of patients as those receiving chemotherapy and RT were also included. The long recruiting period might involve variations in surgical techniques and treatment approach. Moreover, the number of lymph nodes resected in this review was also small. In order to achieve improvement in survival, Herr (4) observed that at least eight and nine lymph nodes should be removed, in node negative and positive patients, respectively. The corresponding figures in our series were respectively eight and seven,. Despite these weaknesses, several prognostic factors, and in particular LND, were found to be predictive of disease specific survival.

An important finding was that $\mathrm{N}$ staging affected disease specific survival in univariate analysis but was replaced by LND in multivariate analysis. Moreover, neither the number of nodes involved nor the number resected affected survival in multivariate analysis. This implies that the concept of surgical margin can also be applied to the lymph node status, and residual disease in the lymph nodes should be avoided. This can be accomplished by a more extensive lymphadenectomy to include clinically undetected lymph node micrometastases and to reduce the LND. However, randomized trials comparing extended and standard lymphadenectomy are needed before concluding that extended lymphadenectomy improves disease specific survival.

The value and extent of pelvic lymphadenectomy during cystectomy were considered controversial in the eighties. Though complete pelvic lymphadenectomy was advocated by some authors (5), others concluded that its contribution to survival was minimal $(6,7)$. There were also proponents of a more limited dissection of pelvic lymph nodes (8). The pendulum swung to the side for meticulous pelvic lymphadenectomy in the nineties (9-11). At the turn of the millennium, data on its morbidity (12) and lymphatic mapping (13-16) were evolving, together with other related concepts like sentinel nodes (15) and LND (4).

Stein (17) introduced the term LND though the concept had been previously mentioned by Herr (18) and Konety (19). He reported that for patients with nodal disease, those with a LND of $20 \%$ or less had a 10 -year recurrence free survival of $43 \%$, compared to $17 \%$ of those with LND more than $20 \%$. We also found in our study that a division line on LND at $20 \%$ discriminated patients with nodal disease into two groups with respect to disease specific survival, with both univariate and multivariate analyses. Sixty four point three percent of those with LND of $20 \%$ or less survived ten years or more, whereas all patients with LND more than $20 \%$ were either dead or censored at 39 months (Figure-8).

Our data showed that LND was a useful prognostic factor supplementary to $\mathrm{N}$ stage for disease specific survival. Future studies are needed before LND can be considered a widely accepted staging system or even used to replace the $\mathrm{N}$ staging. Moreover, the technique of lymphadenectomy, the way of counting the lymph nodes and the histological assessment should be standardized. Lymph node mapping studies (13-16) may facilitate extensive lymphadenectomy allowing, for example, one level higher resection.

Knowing that LND plays a crucial role in determining survival, it is important to define what pre-operative and intra-operative factors will predict LND. This will help to decrease the number of unnecessary cystectomy that will not offer advantage on disease specific survival. We analyzed by logistic regression the effects on LND by several pre-operative and intra-operative factors mentioned above. However, no such factor was found to predict LND in our series.

Our data showed that pT staging affected disease specific survival in univariate and multivariate analyses. This agreed with findings of other series in the literature and our survival figures were comparable to them (1). The marked difference in survival between pT2 and pT3 diseases as shown in Figure-2 may suggest that a more aggressive adjuvant approach should be adopted for extravesical disease.

Other factors that predicted disease specific survival in our series included post-operative RT to distant metastasis, surgical margin and CIS. Postoperative RT to distant metastasis also adversely affected survival in univariate and multivariate analyses. However, this might simply imply that 
those that developed metastases and hence received palliative RT died earlier. A minimal or positive surgical margin was shown to affect survival only in univariate but not in multivariate analysis. This might be because the influence of surgical margin could be explained by other factors such as pT staging, for example. The paradoxical finding of higher disease specific survival associated to CIS just reached statistical significance with univariate analysis ( $\log$ rank, $p=0.0417)$ and not with multivariate analysis.

We also specifically analyzed the effects of pre-operative and post-operative adjuvant therapy. Our results showed that chemotherapy did not alter the survival at all. Raghavan (20) pointed out that multi-center randomized trials had shown survival benefit from neoadjuvant chemotherapy while adjuvant chemotherapy failed to show such benefit. Similarly, our results showed that pre-operative RT to the bladder or post-operative RT to the pelvis did not alter the survival. In this regard, a meta-analysis by Shelley (21) showed a survival benefit with preoperative RT plus radical cystectomy when compared to radical RT plus salvage cystectomy. As mentioned above, our finding that post-operative RT to distant metastasis predicted survival might simply imply that those who developed metastases died earlier.

\section{CONCLUSIONS}

In this retrospective review of radical cystectomy performed for TCC, pT stage, N stage, LND, CIS, surgical margin and post-operative RT to distant metastasis were found to predict disease specific survival. In particular, LND of $20 \%$ discriminated patients into two groups with respect to disease specific survival. The concept of LND may be useful in future staging systems and it shed a light on the importance of extended lymphadenectomy. This review of 133 patients failed to define any preoperative and intra-operative factors that could predict LND. Future studies of larger scale may make this possible so as to decrease the number of unnecessary cystectomy that will not offer advantage on disease specific survival.

\section{CONFLICT OF INTEREST}

\author{
None declared.
}

\section{REFERENCES}

1. Frazier HA, Robertson JE, Dodge RK, Paulson DF: The value of pathologic factors in predicting cancerspecific survival among patients treated with radical cystectomy for transitional cell carcinoma of the bladder and prostate. Cancer. 1993; 71: 3993-4001.

2. Stein JP, Lieskovsky G, Cote R, Groshen S, Feng AC, Boyd S, et al.: Radical cystectomy in the treatment of invasive bladder cancer: long-term results in 1,054 patients. J Clin Oncol. 2001; 19: 666-75.

3. Lerner SP, Skinner DG, Lieskovsky G, Boyd SD, Groshen SL, Ziogas A, et al.: The rationale for en bloc pelvic lymph node dissection for bladder cancer patients with nodal metastases: long-term results. J Urol. 1993; 149: 758-64.

4. Herr HW, Bochner BH, Dalbagni G, Donat SM, Reuter VE, Bajorin DF: Impact of the number of lymph nodes retrieved on outcome in patients with muscle invasive bladder cancer. J Urol. 2002; 167: 1295-8.

5. Lieskovsky G, Skinner DG: Role of lymphadenectomy in the treatment of bladder cancer. Urol Clin North Am. 1984; 11: 709-16.

6. Ariyoshi A, Minoda K, Komatsu K, Fujisawa Y, Yamaguchi A, Yoshida T: Does 'extended' pelvic lymphadenectomy truly contribute to the management of bladder carcinoma? Eur Urol. 1986; 12: 314-7.

7. Herr HW: Bladder cancer: pelvic lymphadenectomy revisited. J Surg Oncol. 1988; 37: 242-5.

8. Wishnow KI, Johnson DE, Ro JY, Swanson DA, Babaian RJ, von Eschenbach AC: Incidence, extent and location of unsuspected pelvic lymph node metastasis in patients undergoing radical cystectomy for bladder cancer. J Urol. 1987; 137: 408-10.

9. Vieweg J, Whitmore WF Jr, Herr HW, Sogani PC, Russo P, Sheinfeld J, et al.: The role of pelvic lymphadenectomy and radical cystectomy for lymph node positive bladder cancer. The Memorial SloanKettering Cancer Center experience. Cancer. 1994; 73 : 3020-8.

10. Turner WH, Markwalder R, Perrig S, Studer UE: Meticulous pelvic lymphadenectomy in surgical treatment of the invasive bladder cancer: an option or a must? Eur Urol. 1998; 33: 21-2. 
11. Leissner J, Hohenfellner R, Thuroff JW, Wolf HK: Lymphadenectomy in patients with transitional cell carcinoma of the urinary bladder; significance for staging and prognosis. BJU Int. 2000; 85: 817-23.

12. Brossner C, Pycha A, Toth A, Mian C, Kuber W: Does extended lymphadenectomy increase the morbidity of radical cystectomy? BJU Int. 2004; 93: 64-6.

13. Leissner J, Ghoneim MA, Abol-Enein H, Thuroff JW, Franzaring L, Fisch M, et al.: Extended radical lymphadenectomy in patients with urothelial bladder cancer: results of a prospective multicenter study. J Urol. 2004; 171: 139-44.

14. Vazina A, Dugi D, Shariat SF, Evans J, Link R, Lerner SP: Stage specific lymph node metastasis mapping in radical cystectomy specimens. J Urol. 2004; 171: 18304.

15. Sherif A, De La Torre M, Malmstrom PU, Thorn M: Lymphatic mapping and detection of sentinel nodes in patients with bladder cancer. J Urol. 2001; 166: 8125.

16. Ghoneim MA, Abol-Enein H: Lymphadenectomy with cystectomy: is it necessary and what is its extent? Eur Urol. 2004; 46: 457-61.
17. Stein JP, Cai J, Groshen S, Skinner DG: Risk factors for patients with pelvic lymph node metastases following radical cystectomy with en bloc pelvic lymphadenectomy: concept of lymph node density. J Urol. 2003; 170:35-41.

18. Herr HW: Superiority of ratio based lymph node staging for bladder cancer. J Urol. 2003; 169: 943-5.

19. Konety BR, Joslyn SA, O'Donnell MA: Extent of pelvic lymphadenectomy and its impact on outcome in patients diagnosed with bladder cancer: analysis of data from the Surveillance, Epidemiology and End Results Program data base. J Urol. 2003; 169: 94650.

20. Raghavan D: Chemotherapy and cystectomy for invasive transitional cell carcinoma of bladder. Urol Oncol. 2003; 21: 468-74.

21. Shelley MD, Wilt TJ, Barber J, Mason MD: A metaanalysis of randomised trials suggests a survival benefit for combined radiotherapy and radical cystectomy compared with radical radiotherapy for invasive bladder cancer: are these data relevant to modern practice? Clin Oncol (R Coll Radiol). 2004; 16: 16671.

Accepted after revision: July 1, 2006

\author{
Correspondence address: \\ Dr. C. W. Cheng \\ Department of Surgery \\ The Chinese University of Hong Kong \\ Prince of Wales Hospital, Hong Kong, China \\ Fax: + 852 2635-9307 \\ E-mail: drmcheng@ hotmail.com
}

\section{EDITORIAL COMMENT}

In this study, the authors have reviewed an experience acquired over the last fourteen years in the management of bladder cancer by radical cystectomy. They looked at the usefulness of the lymph node density as the indicator of prognosis and the disease specific survival. Many factors can influence the outcome of the patients with bladder cancer including $\mathrm{T}$ stage, $\mathrm{N}$ stage and the total number of lymph node retrieved.

A large number of publications have highlighted the importance of prognostic factor including age, gender and lymph node status and 
evidenced that the number of the involved lymph nodes was the single most important prognostic variable.

Herr (1) introduced the term lymph node density indicating the ratio between the number of nodes removed to the number of nodes involved. They found that the ration based lymph node staging which reflect the quality of lymph node dissection was a significant variable prognostic for survival and local control of patients, which are left with node involvement after radical cystectomy.

Stein et al. (2) reported their experience of 244 patients with pathological lymph node metastases treated for primary carcinoma of the bladder. They reported that overall and recurrence free survival were significantly related to the pathological subgroup with the primary bladder tumor. Patients with lymph node

\section{EDITORIAL COMMENT}

The authors present their experience and clinical outcomes in 133 patients who underwent radical cystectomy for transitional cell carcinoma over a 13-year period of time. They found the pT stage and lymph node density and postoperative adjuvant radiotherapy to distant metastases were predictive of cancer specific survival. In addition, in those with lymph node positive disease a $20 \%$ lymph node density was an important risk stratifier. density of $20 \%$ or less had better recurrence free survival when compared to those with more than $20 \%$ (statistically significant). The total number of lymph nodes removed at surgery was also prognostic. Patients with 15 or less lymph nodes removed had 25\% 10-year recurrence-free survival compared with $36 \%$ when greater than 15 lymph nodes were removed.

\section{REFERENCES}

1. Herr H: Superiority of ratio based lymph node staging for bladder cancer. J Urol. 2003; 169: 943-5.

2. Stein JP, Cai J, Groshen S, Skinner DG: Risk factors for patients with pelvic lymph node metastases following radical cystectomy with en bloc pelvic lymphadenectomy: concept of lymph node density. J Urol. 2003; 170: 35-41.

\author{
Dr. Hammad M. Ather \\ Department of Surgery \\ Aga Khan University Hospital \\ Karachi, Pakistan \\ E-mail: hammad.ather@aku.edu
}

The authors comment that radical cystectomy can reach good results with organ confined bladder cancer, which is true, however even those with locally advanced and with regional lymph node involvement benefit from a surgical approach with the best reported outcomes.

The median follow-up is very short and should be noted as potential weakness of the study.

Dr. John Peter Stein Norris Comprehensive Cancer Center University of Southern California Los Angeles, California, USA E-mail: stein@hsc.usc.edu 


\section{EDITORIAL COMMENT}

The purpose of the manuscript was to determine the significance of lymph node density on survival of patients with transitional cell carcinoma of the bladder undergoing cystectomy. There are now 3 or 4 papers demonstrating the significance of lymph node density on recurrence and so this is a relevant paper and topic.

The study design in the most part supports the conclusion; however, the inclusion of patients receiving preoperative and postoperative chemotherapy (21 patients) and the 17 patients receiving preoperative or postoperative radiotherapy to distant metastasis were included.

The main thrust of the paper was to determine the significance of lymph node density on survival. Only 11 patients had a lymph node density $>20 \%$, with 108 presenting lymph node density $<20 \%$. Reaching a conclusion on these 11 patients is concerning.

In conclusion, this is an interesting paper and certainly timely with all the data on the significance of lymph node density.

Dr. Stephen D. Beck

Department of Urology

Indiana University School of Medicine

Indianapolis, Indiana, USA

E-mail:stdbeck@iupui.edu 